\title{
Synthesis, X-Ray Crystal Structures, Biological Evaluation, and Molecular Docking Studies of a Series of Barbiturate Derivatives
}

\author{
Assem Barakat, ${ }^{1,2}$ Hazem A. Ghabbour, ${ }^{3,4}$ Abdullah Mohammed Al-Majid, ${ }^{1}$ \\ Qurat-ul-ain, ${ }^{5}$ Rehan Imad, ${ }^{5}$ Kulsoom Javaid, ${ }^{5}$ Nimra Naveed Shaikh, ${ }^{5}$ Sammer Yousuf, ${ }^{5}$ \\ M. Iqbal Choudhary, ${ }^{1,5}$ and Abdul Wadood ${ }^{6}$ \\ ${ }^{1}$ Department of Chemistry, College of Science, King Saud University, P.O. Box 2455, Riyadh 11451, Saudi Arabia \\ ${ }^{2}$ Department of Chemistry, Faculty of Science, Alexandria University, P.O. Box 426, Ibrahimia, Alexandria 21321, Egypt \\ ${ }^{3}$ Department of Pharmaceutical Chemistry, College of Pharmacy, King Saud University, P.O. Box 2457, Riyadh 11451, Saudi Arabia \\ ${ }^{4}$ Department of Medicinal Chemistry, Faculty of Pharmacy, Mansoura University, Mansoura 35516, Egypt \\ ${ }^{5}$ H.E.J. Research Institute of Chemistry, International Center for Chemical and Biological Sciences, University of Karachi, \\ Karachi 75270, Pakistan \\ ${ }^{6}$ Department of Biochemistry, Abdul Wali Khan University, Mardan 23200, Pakistan
}

Correspondence should be addressed to Assem Barakat; ambarakat@ksu.edu.sa

Received 4 February 2016; Revised 6 April 2016; Accepted 10 April 2016

Academic Editor: Gustavo Portalone

Copyright (C) 2016 Assem Barakat et al. This is an open access article distributed under the Creative Commons Attribution License, which permits unrestricted use, distribution, and reproduction in any medium, provided the original work is properly cited.

A series of barbiturates derivatives synthesized and screened for different set of bioassays are described. The molecular structures of compounds $\mathbf{5 a}, \mathbf{5 d}$, and $\mathbf{5 f}$ were solved by single-crystal X-ray diffraction techniques. The results of bioassay show that compounds $\mathbf{4 a}, \mathbf{4 b}, \mathbf{4 c}, \mathbf{4 d}, \mathbf{4 e}, \mathbf{4 f}$, and $\mathbf{4 g}$ are potent antioxidants in comparison to the tested standards, butylated hydroxytoluene (BHT), and $N$ acetylcysteine. Compounds $\mathbf{4 a}-\mathbf{4 e}\left(\mathrm{IC}_{50}=101.8 \pm 0.8-124.4 \pm 4.4 \mu \mathrm{M}\right)$ and $\mathbf{4 g}\left(\mathrm{IC}_{50}=104.1 \pm 1.9 \mu \mathrm{M}\right)$ were more potent antioxidants than the standard $\left(\mathrm{BHT}, \mathrm{IC}_{50}=128.8 \pm 2.1 \mu \mathrm{M}\right)$. The enzyme inhibition potential of these compounds was also evaluated, in vitro, against thymidine phosphorylase, $\alpha$-glucosidase, and $\beta$-glucuronidase enzymes. Compounds $\mathbf{4 c}, \mathbf{4 h}, \mathbf{4 o}, \mathbf{4 p}, \mathbf{4 q}, \mathbf{5 f}$, and $\mathbf{5 m}$ were found to be potent $\alpha$-glucosidase inhibitors and showed more activity than the standard drug acarbose, whereas compounds $4 \mathbf{v}$, and $\mathbf{5 h}$ were found to be potent thymidine phosphorylase inhibitors, more active than the standard drug, 7-deazaxanthine. All barbiturates derivatives $(\mathbf{4 a}-\mathbf{4 x}, \mathbf{4 z}$, and $\mathbf{5 a}-\mathbf{5 m})$ were found to be noncytotoxic against human prostate (PC-3), Henrietta Lacks cervical (HeLa) and Michigan Cancer Foundation-7 breast (MCF-7) cancer cell lines, and 3T3 normal fibroblast cell line, except 4y which was cytotoxic against all the cell lines.

\section{Introduction}

Derivatives of 1,3-dimethylbarbituric acid including pyrimidine-2,4,6-trione (PYT) heterocycles play an important role in the pharmaceutical chemistry $[1,2]$. Barbituric acid and its derivatives are well known as hypotensive, antibacterial, and antisclerotic, hypnotic, sedative, anticonvulsant, antispasmodic, anticancer, matrix metalloproteinase inhibitors, anticonvulsants, and anti-inflammatory and anxiolytic agents, as well as being used in local anesthetic drugs [2-9]. Barbituric acid derivatives are also known for their use as clinical drugs, such as Seconal, Veronal, phenobarbital, bucolome, and sodium pentothal (Figure 1, 1-5) [10-13]. The interesting mechanism of action of barbital and phenobarbital against epilepsy made them attractive targets for medicinal chemists. They have also been investigated as anticancer and antiAIDS agents [14]. Reactive oxygen species (ROS) are oxygencentered free radicals such as reactive oxygen species (ROS) which are peroxyls $\left(\mathrm{ROO}^{\circ}\right)$, superoxides $\left(\mathrm{O}_{2}{ }^{--}\right)$, hydroxyls $\left(\mathrm{HO}^{\circ}\right)$, alkoxyls $\left(\mathrm{RO}^{\circ}\right)$, and nitric oxides $\left(\mathrm{NO}^{\circ}\right)$ [15], while hypochlorous acid ( $\mathrm{HOCl})$, hydrogen peroxide $\left(\mathrm{H}_{2} \mathrm{O}_{2}\right)$, and singlet oxygen $\left(\mathrm{O}_{2}\right)$ are classified as nonradical ROS. The ROS may react in various environments [15]. However overproduction of ROS species has been linked to cell membrane 
<smiles>CCCCc1c(O)n(-c2ccccc2)c(=O)[nH]c1=O</smiles>

1 (Bucolome)<smiles>CCCC(C)C1(CC)C(=O)N=C([Se])NC1=O</smiles>

2 (Sodium pentothal)

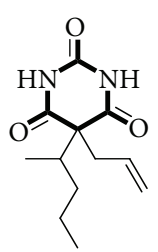

3 (Seconal)

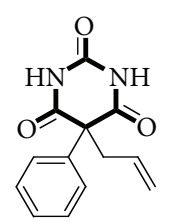

4 (Phenobarbital)

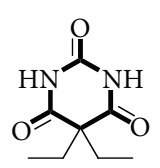

5 (Veronal)<smiles>CCOc1cc(C=C2C(=O)NC(=O)N(Cc3ccccc3)C2=O)ccc1O</smiles>

6 (Hsp90 inhibitor)<smiles>O=C1NC(=O)C(c2ccc(-c3ccccc3)cc2)(N2CCN(c3ccc([N+](=O)[O-])cc3)CC2)C(=O)N1</smiles>

7 (Ro 28-2653, MMP inhibitor)<smiles>O=C1NC(=O)C(C2c3cc(Cl)ccc3Oc3[nH]c(=O)[nH]c(=O)c32)C(=O)N1</smiles>

8 (EM20-25, BCL-2 inhibitor)<smiles>CCCCOc1c(C(c2ccc([N+](=O)[O-])cc2)c2c(OO[NH3+])n(C)c(=O)n(C)c2=O)c(=O)n(C)c(=O)n1C</smiles>

Potent nitric oxide scavenger $10: \mathrm{IC}_{50}=69 \pm 1.66 \mu \mathrm{M}$ Std ascorbic acid $\mathrm{IC}_{50}=618 \pm 2.0 \mu \mathrm{M}$

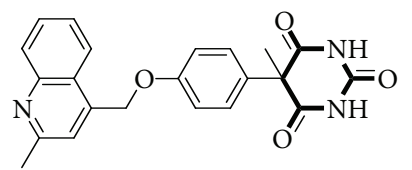

9 (TACE inhibitor)

FIGURE 1: Structure of biologically active barbituric acid derivatives.

damage, protein and enzyme modifications, and DNA damage within the body [16]. These types of changes accelerate aging and play a causative role in a variety of degenerative diseases, such as cancers, atherosclerosis, brain disfunction, inflammation, shock, and heart diseases. Recently Barakat et al. [17] have synthesized some novel salts of Michael adduct derived from PYT and evaluated them for NO (nitric oxide) scavenging activity. Compound $\mathbf{1 0}$ was found to possess several hundredfold more activity against nitric oxide radical $\left(\mathrm{IC}_{50}\right.$ values of $\left.69 \pm 1.66 \mu \mathrm{M}\right)$ than the standard drug ascorbic acid $\left(\mathrm{IC}_{50}=618 \pm 2.0 \mu \mathrm{M}\right)$.

Because of the important biological activities, PYT is widely used as a synthon in the design of antitumor agents. It has a high efficacy to form hydrogen bonding with drug targets (6-9, Figure 1). Singh et al. have reported the activity of a series of new $N$-benzyl indole-pyrimidine-2,4,6-trione hybrids against a panel of 60 human tumor cell lines. Several of these analogs were also found to be inhibitors of DNA repair and replication stress response polymerases [18].

The discovery and development of effective antioxidants capable of supplementing body's antioxidant system is an important area of health care research. Similarly research efforts are focused on the identification of effective and safe inhibitors of $\alpha$-glucosidase as potential antidiabetic agents.

The focus in recent years is on the development of resource-efficient, environmentally benign, and sustainable chemistry practices. In continuation of our work in this field, we describe here the synthesis of a series of previously reported $[17,19,20]$ and new PYT adducts. These compounds were evaluated for their antioxidant activity by using DDPH scavenging assay, as well as for in vitro enzyme inhibition activities against alpha-glucosidase, thymidine phosphorylase, and $\beta$-glucuronidase enzymes.

\section{Materials and Methods}

The chemical reagents used for the preparation of the target compounds were commercially available and used without further purification. ${ }^{1} \mathrm{H}$-NMR and ${ }^{13} \mathrm{C}$-NMR were measured using Bruker Avance AV-600 NMR spectrometer, MS were carried out with a Jeol, JMS- $600 \mathrm{H}$ instrument, and singlecrystal X-ray diffraction was collected using a Bruker SMART APEX II CCD diffractometer.

2.1. General Procedure for Aldol Condensation Michael Addition for the Synthesis of $\mathbf{4}$ and $\mathbf{5}$ (GP1). A mixture of aldehyde $3(1.5 \mathrm{mmol})$ and 1 and $2(3 \mathrm{mmol})$ as well as $\mathrm{Et}_{2} \mathrm{NH}(1.5 \mathrm{mmol}$, $155 \mu \mathrm{L}$ ) in $3 \mathrm{~mL}$ of degassed $\mathrm{H}_{2} \mathrm{O}$ (bubbling nitrogen through the water) was stirred at room temperature for $1-5 \mathrm{~h}$ until TLC showed complete disappearance of the reactants. The precipitate was removed by filtration and washed with ether $(3 \times 20 \mathrm{~mL})$. The solid product was dried to afford pure products 4 and 5 .

The full characterization of the synthesized compounds 4 and $\mathbf{5}$ and the X-ray crystal data of $\mathbf{5 a}, \mathbf{5 d}$, and $\mathbf{5 f}$ can be seen in the Supplementary Materials (see supplementary materials available online at http://dx.doi.org/10.1155/2016/8517243).

2.2. Biological Activity. The samples of the synthesized compound were screened against DPPH radical, $\beta$-glucuronidase 


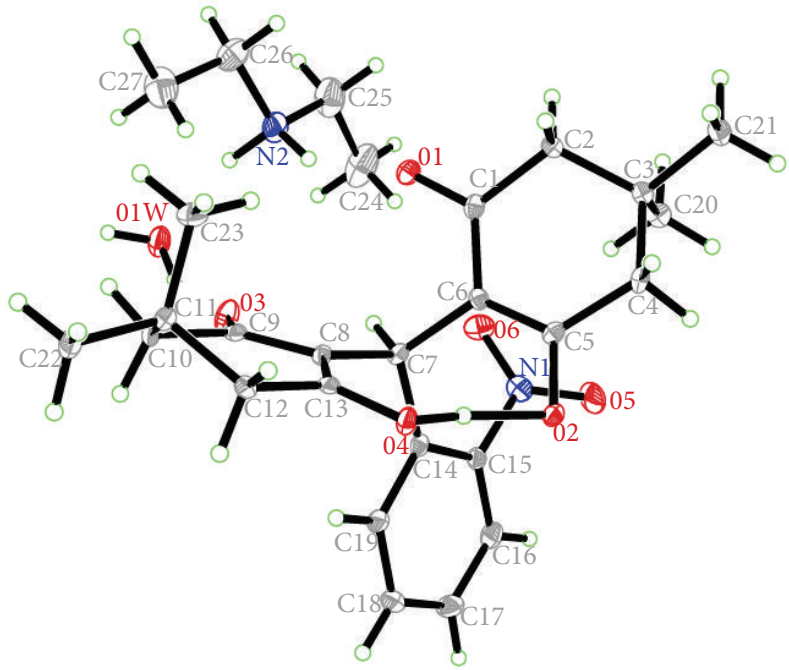

$5 \mathbf{a}$

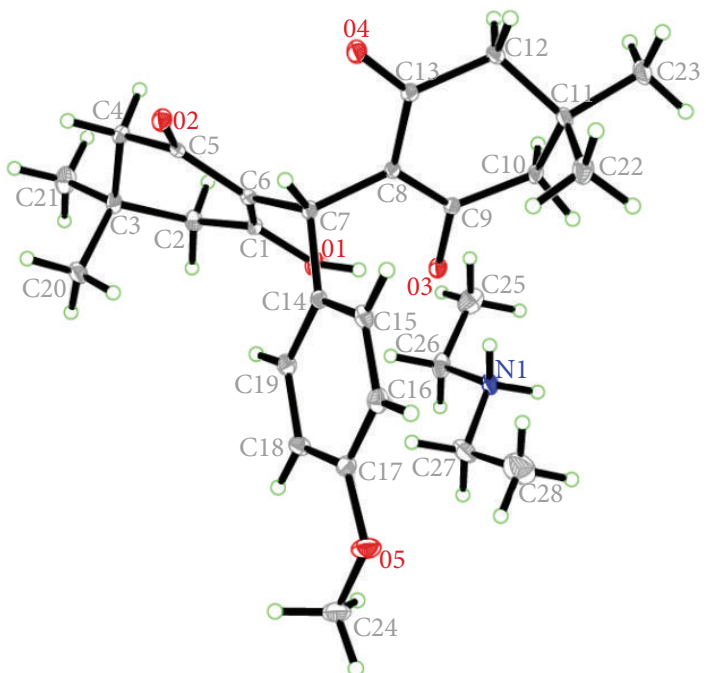

$5 d$

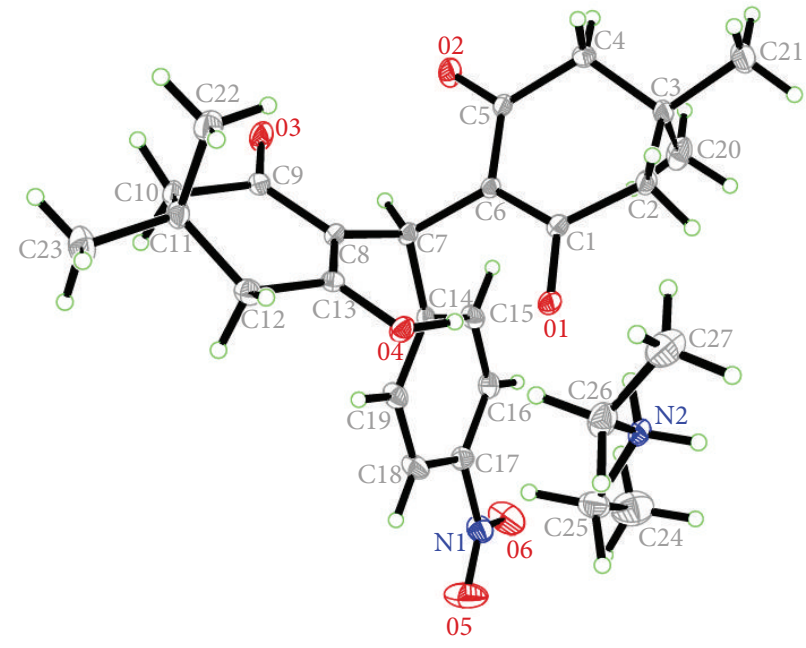

$5 f$

Figure 2: ORTEP diagram of $\mathbf{5 a}, \mathbf{5 d}$, and $\mathbf{5 f}$. Displacement ellipsoids are plotted at the $40 \%$ probability level for non- $\mathrm{H}$ atoms.

inhibition assay, thymidine phosphorylase inhibition assay, and $\alpha$-glucosidase inhibition assay and full methods descriptions are provided in Supplementary Materials.

\section{Results and Discussion}

3.1. Chemistry. We have been focusing on the development of highly expedient methods for the synthesis of diverse heterocyclic compounds of biological importance via onepot multicomponent reactions (MCRs) and avoiding organic solvents in organic synthesis $[17,19,20]$. This had led to the development of several efficient, environmentally benign, clean, and economical process technology (Green Chemistry Concepts). In current reaction strategy, equimolar amounts of barbituric acid (1) and dimedone (2) with aldehyde (3) in the presence of aqueous diethylamine medium at RT yielded salts of PYT adducts $\mathbf{4 a}-\mathbf{4 y}$ and $\mathbf{5 a}-\mathbf{5 m}$ were obtained in quantitative yields by simple filtration [17-20].
Products $4 \mathbf{s}$, $4 \mathbf{t}$, and $\mathbf{5 g}$ were identified as new products (Scheme 1).

3.2. X-Ray Diffraction. The molecular structures of compounds $\mathbf{5 a}, \mathbf{5 d}$, and $\mathbf{5 f}$ were unambiguously deduced by single-crystal X-ray diffraction technique (Figure 2). The crystals of the synthesized molecules $\mathbf{5 a}, \mathbf{5 d}$, and $\mathbf{5 f}$ were grown by slow diffusion of diethyl ether solution of pure samples 5a, 5d, and $\mathbf{5 f}$ in DCM/EtOH at room temperature followed by allowing standing for $24 \mathrm{~h}$. The structures were resolved by direct methods by using the SHELXL97 program $[21,22]$ in the SHELXTL-plus package and refined by a full-matrix least-squares procedure on $F^{2}$ using SHELXS97. Diffraction data were collected on a Bruker APEX-II CCD diffractometer (Bruker AXS GmbH). The crystal structure and refinement data of compounds $\mathbf{5 a}, \mathbf{5 d}$, and $\mathbf{5} \mathbf{f}$ are listed in Table 1. The final atomic coordinates for all atoms and complete listing of bond distance and angles are presented 


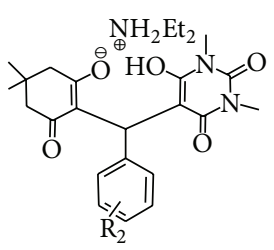

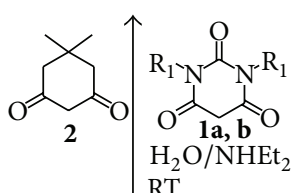

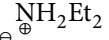

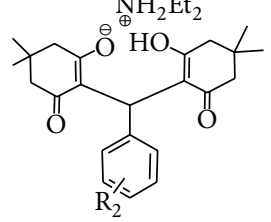

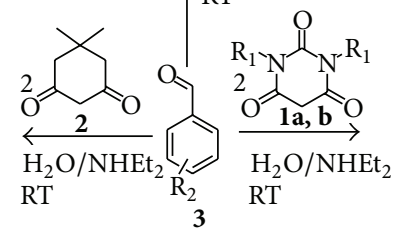

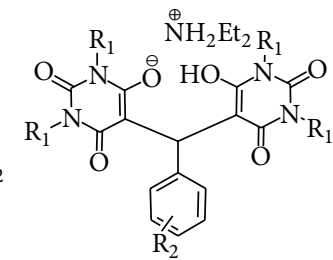<smiles>[R]c1ccc(C(c2c(O)n(C)c(=O)n(C)c2=O)C2C(=O)N(C)C(=O)N2C)cc1[R]</smiles>

4a $\mathrm{R}=\mathrm{CHO}, \mathrm{R}_{1}=\mathrm{H}$

$4 \mathbf{b} \mathrm{R}=\mathrm{H}, \mathrm{R}_{1}=\mathrm{CH}_{3}$

4c $\mathrm{R}=\mathrm{NO}_{2}, \mathrm{R}_{1}=\mathrm{H}$

4d $\mathrm{R}=\mathrm{OCH}_{3}, \mathrm{R}_{1}=\mathrm{H}$

4e $\mathrm{R}=\mathrm{H}, \mathrm{R}_{1}=\mathrm{Br}$

4f $\mathrm{R}=\mathrm{OH}, \mathrm{R}_{1}=\mathrm{H}$

$4 \mathrm{~g} \mathrm{R}=\mathrm{CH}_{3}, \mathrm{R}_{1}=\mathrm{H}$

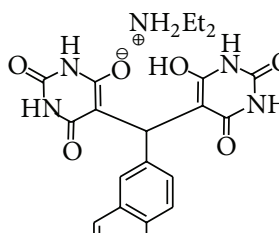

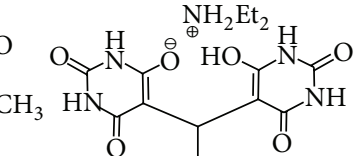

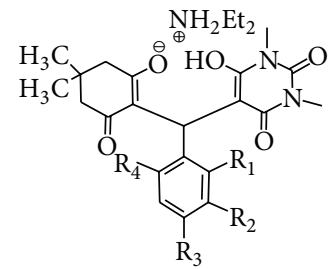

$4 \mathrm{~m}_{1}=\mathrm{R}_{2}=\mathrm{R}_{3}=\mathrm{R}_{4}=\mathrm{H}$

4n $\mathrm{R}_{1}=\mathrm{R}_{2}=\mathrm{R}_{4}=\mathrm{H}, \mathrm{R}_{3}=\mathrm{CH}_{3}$

4o $\mathrm{R}_{1}=\mathrm{R}_{2}=\mathrm{R}_{4}=\mathrm{H}, \mathrm{R}_{3}=\mathrm{OCH}_{3}$

$4 p \mathrm{R}_{1}=\mathrm{R}_{2}=\mathrm{R}_{4}=\mathrm{H}, \mathrm{R}_{3}=\mathrm{Cl}$

$4 \mathbf{q} \mathrm{R}_{1}=\mathrm{R}_{2}=\mathrm{R}_{4}=\mathrm{H}, \mathrm{R}_{3}=\mathrm{Br}$

$4 \mathbf{r} \mathrm{R}_{1}=\mathrm{R}_{3}=\mathrm{R}_{4}=\mathrm{H}, \mathrm{R}_{2}=\mathrm{Br}$

4s $\mathrm{R}_{1}=\mathrm{NO}_{2}, \mathrm{R}_{2}=\mathrm{R}_{3}=\mathrm{R}_{4}=\mathrm{H}$

4t $\mathrm{R}_{1}=\mathrm{R}_{2}=\mathrm{R}_{4}=\mathrm{H}, \mathrm{R}_{3}=\mathrm{NMe}_{2}$

$4 \mathbf{v} \mathrm{R}_{1}=\mathrm{R}_{2}=\mathrm{R}_{4}=\mathrm{H}, \mathrm{R}_{3}=\mathrm{OH}$

$4 \mathrm{w} \mathrm{R}_{1}=\mathrm{R}_{3}=\mathrm{Cl}, \mathrm{R}_{2}=\mathrm{R}_{4}=\mathrm{H}$

$4 \mathrm{R}_{1}=\mathrm{R}_{2}=\mathrm{R}_{4}=\mathrm{H}, \mathrm{R}_{3}=\mathrm{CHO}$

4y $\mathrm{R}_{1}=\mathrm{R}_{4}=\mathrm{Cl}, \mathrm{R}_{2}=\mathrm{R}_{3}=\mathrm{H}$

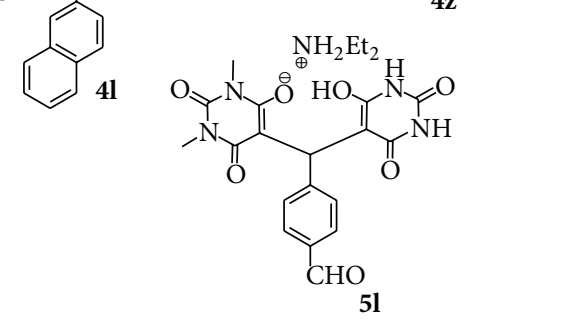<smiles>CCCCN1C(=O)C(C(c2ccc3ccccc3c2)c2c(O)n(C)c(=O)n2C)C(=O)N1C</smiles>

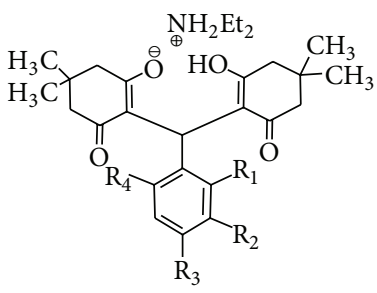

5a $\mathrm{R}_{1}=\mathrm{NO}_{2}, \mathrm{R}_{2}=\mathrm{R}_{3}=\mathrm{R}_{4}=\mathrm{H}$

5b $\mathrm{R}_{1}=\mathrm{R}_{3}=\mathrm{R}_{4}=\mathrm{H}, \mathrm{R}_{2}=\mathrm{CH}_{3}$

$5 c \mathrm{R}_{1}=\mathrm{R}_{3}=\mathrm{R}_{4}=\mathrm{CH}_{3}, \mathrm{R}_{2}=\mathrm{H}$

$5 \mathrm{~d} \mathrm{R}_{1}=\mathrm{R}_{2}=\mathrm{R}_{4}=\mathrm{H}, \mathrm{R}_{3}=\mathrm{OCH}_{3}$

5e $\mathrm{R}_{1}=\mathrm{R}_{4}=\mathrm{Cl}, \mathrm{R}_{2}=\mathrm{R}_{3}=\mathrm{H}$

5 f $\mathbf{R}_{1}=\mathrm{R}_{2}=\mathrm{R}_{4}=\mathrm{H}, \mathrm{R}_{3}=\mathrm{NO}_{2}$

$5 \mathrm{~g} \mathrm{R}_{1}=\mathrm{R}_{2}=\mathrm{R}_{4}=\mathrm{H}, \mathrm{R}_{3}=\mathrm{CHO}$

5 h $\mathrm{R}_{1}=\mathrm{R}_{2}=\mathrm{R}_{4}=\mathrm{H}, \mathrm{R}_{3}=\mathrm{OH}$

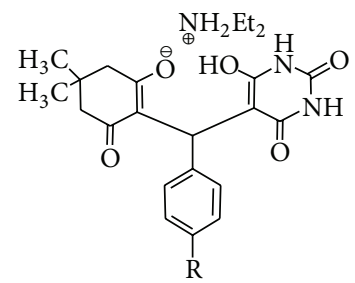

$5 \mathbf{i} \mathrm{R}=\mathrm{Cl}$

5j $\mathrm{R}=\mathrm{H}$

$5 \mathrm{k} \mathrm{R}=\mathrm{Br}$

$51 \mathrm{R}=\mathrm{CH}_{3}$

$5 \mathrm{~m} \mathrm{R}=\mathrm{CHO}$

SCHEme 1: Synthesis of the target compounds. 
TABLE 1: The crystal and experimental data of compounds $\mathbf{5 a}, \mathbf{5} \mathbf{d}$, and $\mathbf{5 f}$.

\begin{tabular}{|c|c|c|c|}
\hline & $5 a$ & $5 d$ & $5 f$ \\
\hline Empirical formula & $\mathrm{C}_{23} \mathrm{H}_{26} \mathrm{NO}_{6} \cdot \mathrm{C}_{4} \mathrm{H}_{12} \mathrm{~N} \cdot \mathrm{H}_{2} \mathrm{O}$ & $\mathrm{C}_{24} \mathrm{H}_{29} \mathrm{O}_{5} \cdot \mathrm{C}_{4} \mathrm{H}_{12} \mathrm{~N}$ & $\mathrm{C}_{23} \mathrm{H}_{26} \mathrm{NO}_{6} \cdot \mathrm{C}_{4} \mathrm{H}_{12} \mathrm{~N}$ \\
\hline Formula weight & 504.61 & 471.62 & 486.59 \\
\hline Temperature & $100 \mathrm{~K}$ & $100 \mathrm{~K}$ & $100 \mathrm{~K}$ \\
\hline Wavelength & Mo $K \alpha$ radiation, $\lambda=0.71073 \AA$ & Mo $K \alpha$ radiation, $\lambda=0.71073 \AA$ & Mo $K \alpha$ radiation, $\lambda=0.71073 \AA$ \\
\hline Crystal system & Orthorhombic & Monoclinic & Monoclinic \\
\hline Space group & $P 2_{1} 2_{1} 2_{1}$ & $P 2_{1} / n$ & $P 2_{1} / n$ \\
\hline$a$ & $11.6155(5) \AA$ & $10.2288(5) \AA$ & $10.1137(4) \AA$ \\
\hline$b$ & $12.0514(5) \AA$ & $18.0048(7) \AA$ & 18.1225 (7) $\AA$ \\
\hline$c$ & $19.3665(7) \AA$ & $15.0893(7) \AA$ & $15.4853(6) \AA$ \\
\hline$\beta$ & $90.00^{\circ}$ & $106.425(2)^{\circ}$ & $106.730(1)^{\circ}$ \\
\hline Volume & $2710.98(19) \AA^{3}$ & $2665.6(2) \AA^{3}$ & $2718.09(18) \AA^{3}$ \\
\hline$Z$ & 4 & 4 & 4 \\
\hline Calculated density & $1.236 \mathrm{Mg} \mathrm{m}^{-3}$ & $1.175 \mathrm{Mg} \mathrm{m}^{-3}$ & $1.189 \mathrm{Mg} \mathrm{m}^{-3}$ \\
\hline Absorption coefficient & $0.09 \mathrm{~mm}^{-1}$ & $0.08 \mathrm{~mm}^{-1}$ & $0.08 \mathrm{~mm}^{-1}$ \\
\hline$F(000)$ & 1088 & 1024 & 1048 \\
\hline Crystal size & $0.57 \times 0.41 \times 0.07 \mathrm{~mm}$ & $0.66 \times 0.33 \times 0.25 \mathrm{~mm}$ & $0.33 \times 0.29 \times 0.22 \mathrm{~mm}$ \\
\hline$\theta$ range & $2.4-24.2^{\circ}$ & $2.2-29.7^{\circ}$ & $2.2-32.1^{\circ}$ \\
\hline Reflections collected & 28531 & 67389 & 25488 \\
\hline Reflections unique & 4501 & 6194 & 4829 \\
\hline Independent reflections & 6224 & 11203 & 6233 \\
\hline Parameters & 350 & 326 & 334 \\
\hline$R_{\text {int }}$ & 0.090 & 0.122 & 0.053 \\
\hline$R\left[F^{2}>2 \sigma\left(F^{2}\right)\right]$ & 0.054 & 0.069 & 0.055 \\
\hline$w R\left(F^{2}\right)$ & 0.109 & 0.156 & 0.132 \\
\hline Goodness of fit & 1.05 & 1.01 & 1.09 \\
\hline $\operatorname{Max} / \min \rho$ e $\AA^{-3}$ & 0.42 and -0.27 & 0.43 and -0.30 & 0.29 and -0.26 \\
\hline CCDC number & 1444058 & 1443783 & 1444050 \\
\hline
\end{tabular}

in supplementary information. ORTEP drawings of final Xray model of compounds $\mathbf{5 a}, \mathbf{5} \mathbf{d}$, and $\mathbf{5} \mathbf{f}$ with the atomic numbering scheme are presented in Figure 2, while crystal packing presentation of compounds $\mathbf{5 a}, \mathbf{5 d}$, and $\mathbf{5 f}$ is shown in Figure 3.

Compound 5a crystallizes in orthorhombic crystal system in a space group of $P 22_{1} 2_{1}$; on the other hand, compounds $\mathbf{5} \mathbf{d}$ and $\mathbf{5} \mathbf{f}$ crystalize in monoclinic crystal system in a space group of $P 2_{1} / n$. It was observed that the compound 5 a has hydrogen bonding between the $\mathrm{C}_{5}-\mathrm{O}_{2} \cdots \mathrm{H} \cdots \mathrm{O}_{4}-\mathrm{C}_{13}$, while compounds $\mathbf{5} \mathbf{d}$ and $\mathbf{5} \mathbf{f}$ exist only in the enol form. There are two strong intramolecular hydrogen bonds on compound 5a between $\mathrm{O}_{4}-\mathrm{H}_{1} \mathrm{O}_{4} \cdots \mathrm{O}_{2}$ and $\mathrm{N}_{2}-\mathrm{H}_{2} \mathrm{~N}_{2} \cdots \mathrm{O}_{1}$, while compounds $\mathbf{5 d}$ and $\mathbf{5 f}$ have no intramolecular hydrogen bonding. The crystal structures of the tested compounds 5a, $\mathbf{5 d}$, and $\mathbf{5} \mathbf{f}$ were formed by different hydrogen bonds between the molecules and diethyl amine and water solvent molecules in case of $\mathbf{5 a}$ to form network structures. Crystallographic data for the solved structures $\mathbf{5 a}, \mathbf{5 d}$, and $\mathbf{5 f}$ were deposited to the Cambridge Crystallographic Data Center as supplementary publication numbers CCDC-1444058, CCDC-1443783, and CCDC-1444050, respectively, which can be obtained free of charge from the Cambridge Crystallographic Data Centre via http://www.ccdc.cam.ac.uk/data_request/cif.
3.3. Biological Activity Evaluation. All synthesized diethyl ammonium salts of barbiturates having bis(6-hydroxy-1,3dimethyl-2,4-dioxo-1,2,3,4-tetrahydropyrimidin-5-yl) (4a4h), bis (6-hydroxypyrimidine-2,4(1H,3H)-dione (4i-4l), and (2-hydroxy-4,4-dimethyl-6-oxocyclohex-1-en-1-yl)-1,3-dimethyl-2,6-dioxo-1,2,3,6-tetrahydropyrimidin-4-olate (4m$\mathbf{4 z}$ ) as well as bis(2-hydroxy-4,4-dimethyl-6-oxocyclohex1-ene) $(\mathbf{5} \mathbf{a}-\mathbf{5 m})$ as basic nuclei were screened for their antioxidant potential in vitro by using $\mathrm{DPPH}$ radical scavenging assay and cytotoxicity against PC-3, HeLa, and MCF-7 cancer cell lines and 3T3 normal fibroblast cell lines. These derivatives $\mathbf{4 a}-\mathbf{4 z}$ and $\mathbf{5 a}-\mathbf{5 m}$ were also evaluated for in vitro enzyme inhibition activities against $\alpha$-glucosidase, thymidine phosphorylase, and $\beta$-glucuronidase enzymes. The results are summarized in Table 2 .

3.3.1. Antioxidant Activity (DPPH Radical Scavenging Assay). Among series of compounds $(\mathbf{4 a}-\mathbf{4 h})$ having bis(6-hydroxy1,3-dimethyl-2, 4-dioxo-1, 2, 3, 4-tetrahydropyrimidin-5-yl) ring as basic nucleus, compounds $4 \mathbf{a}\left(\mathrm{IC}_{50}=118.8 \pm 2.1 \mu \mathrm{M}\right)$, 4b $\left(\mathrm{IC}_{50}=117 \pm 2.4 \mu \mathrm{M}\right), 4 \mathbf{c}\left(\mathrm{IC}_{50}=111.9 \pm 1.9 \mu \mathrm{M}\right), \mathbf{4 d}$ $\left(\mathrm{IC}_{50}=124.4 \pm 4.4 \mu \mathrm{M}\right), \mathbf{4 e}\left(\mathrm{IC}_{50}=101.1 \pm 0.8 \mu \mathrm{M}\right), \mathbf{4 f}$ $\left(\mathrm{IC}_{50}=148.6 \pm 2.6 \mu \mathrm{M}\right)$, and $4 \mathrm{~g}\left(\mathrm{IC}_{50}=104.1 \pm 1.9 \mu \mathrm{M}\right)$ were found to be potent antioxidants in comparison to the 


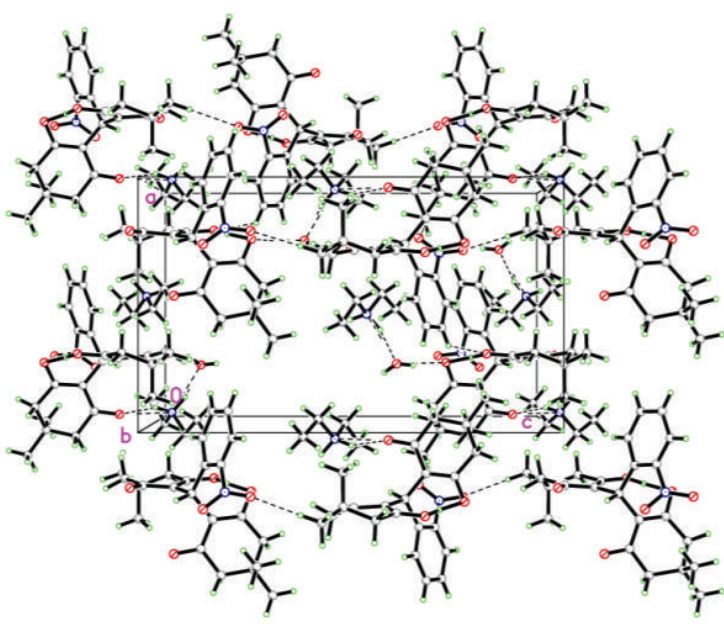

$5 \mathbf{a}$

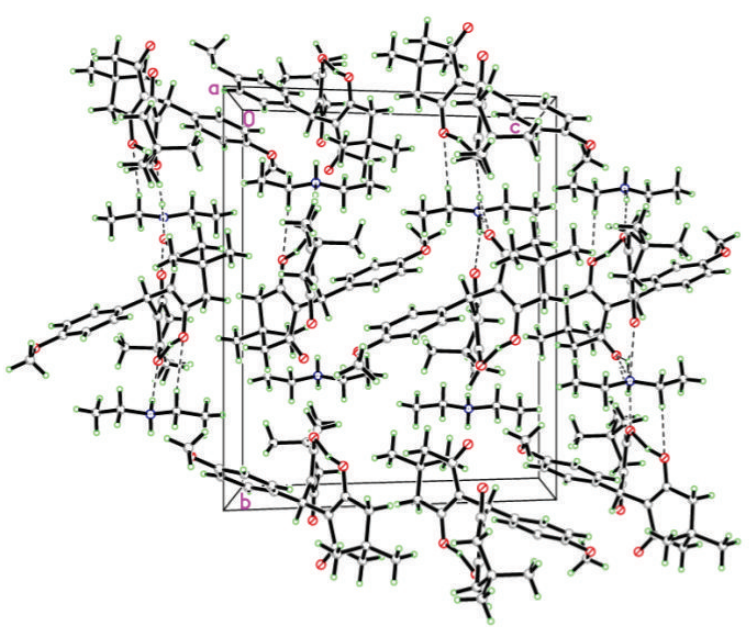

$5 d$

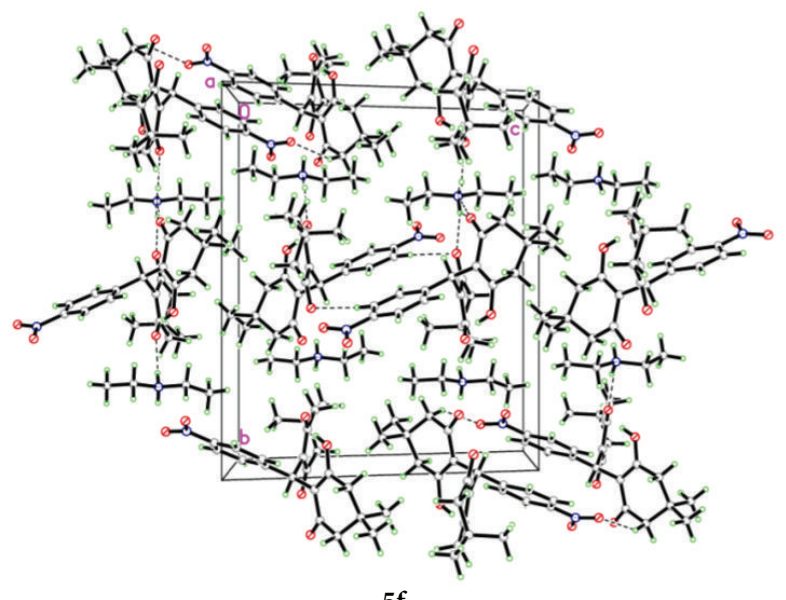

$5 f$

FIGURE 3: Molecular packing of 5a, 5d, and $\mathbf{5 f}$ viewed approximately three-dimensional network, along $b$ axis and three-dimensional network, respectively. Intermolecular interaction is drawn as dashed lines.

standards BHT $\left(\mathrm{IC}_{50}=128.8 \pm 2.1 \mu \mathrm{M}\right)$ and $N$-acetylcysteine $\left(\mathrm{IC}_{50}=107.6 \pm 2.8 \mu \mathrm{M}\right)$. Compounds $4 \mathrm{a}-4 \mathrm{e}\left(\mathrm{IC}_{50}=\right.$ $101.8 \pm 0.8-124.4 \pm 4.4 \mu \mathrm{M})$ were found to be more active compared to the standard BHT $\left(\mathrm{IC}_{50}=128.8 \pm 2.1 \mu \mathrm{M}\right)$. The meta-bromo-substituted phenyl ring containing compound $4 \mathbf{e}\left(\mathrm{IC}_{50}=101.1 \pm 0.8 \mu \mathrm{M}\right)$ was the most potent member of the series (4a-4e), followed by the para-methyl-substituted phenyl ring containing compound $\mathbf{4 g}\left(\mathrm{IC}_{50}=104.1 \pm 1.9 \mu \mathrm{M}\right)$. A gradual decrease in activity was observed for compounds 4c $\left(\mathrm{IC}_{50}=119.9 \pm 1.9 \mu \mathrm{M}\right)$ and $4 \mathbf{a}\left(\mathrm{IC}_{50}=118.8 \pm 3.1 \mu \mathrm{M}\right)$ having para-nitro- and para-aldehyde-substituted phenyl ring attached to central bis(6-hydroxy-1,3-dimethyl-2,4dioxo-1,2,3,4-tetrahydropyrimidin-5-yl) ring. A decrease in $\mathrm{DPPH}$ radical scavenging activity was due to positional effect of methyl substituent on phenyl ring which was observed for compound $4 \mathbf{b}\left(\mathrm{IC}_{50}=117 \pm 3.1 \mu \mathrm{M}\right)$ having meta-substituted phenyl ring instead of para-methyl-substituted phenyl ring $\left(4 \mathrm{~g}, \mathrm{IC}_{50}=104.1 \pm 1.9 \mu \mathrm{M}\right)$. The electron donating parahydroxyl-substituted phenyl ring containing compound $\mathbf{4 f}$ $\left(\mathrm{IC}_{50}=148.6 \pm 2.6 \mu \mathrm{M}\right)$ was found to be a last active member of the series. However, a considerable increase in activity was observed for compound $4 \mathbf{d}\left(\mathrm{IC}_{50}=124.4 \pm 4.4 \mu \mathrm{M}\right)$ having a para-methoxy group instead of hydroxyl functionality on phenyl ring, whereas naphthalene ring containing barbiturate $4 \mathbf{h}\left(\mathrm{IC}_{50}=133.6 \pm 5.7 \mu \mathrm{M}\right)$ was also found to be a weak antioxidant agent against DPPH radicals.

Among second series of the tested compounds 4i-4l having bis(6-hydroxypyrimidine-2,4 $(1 \mathrm{H}, 3 \mathrm{H})$-dione as backbone, compounds having para-methyl- $\left(4 \mathbf{i}, \mathrm{IC}_{50}=384.9 \pm\right.$ $6.6 \mu \mathrm{M})$, para-chloro- $\left(4 \mathbf{j}, \mathrm{IC}_{50}=384.3 \pm 8.8 \mu \mathrm{M}\right)$, and paramethoxy- $\left(41, \mathrm{IC}_{50}=397.9 \pm 0.9 \mu \mathrm{M}\right)$ substituted phenyl ring attached to the central bis(6-hydroxypyrimidine-2,4(1H,3H)dione) showed better antioxidant activity as compared to BHT $\left(\mathrm{IC}_{50}=128.8 \pm 2.1 \mu \mathrm{M}\right)$ and $N$-acetylcysteine $\left(\mathrm{IC}_{50}=\right.$ $107.6 \pm 2.8 \mu \mathrm{M})$, whereas naphthalene containing compound 41 was found to be inactive.

(2-Hydroxy-4,4-dimethyl-6-oxocyclohex-1-en-1-yl)-1,3dimethyl-2,6-dioxo-1,2,3,6-tetrahydropyrimidin-4-olate ring containing compounds $\mathbf{4 m}-\mathbf{4 z}$ were also evaluated for their antioxidant potential in vitro in DPPH radical scavenging assay. These compounds were found to be significant to weak inhibitors of free radicals $\left(\mathrm{IC}_{50}=133.2 \pm 4.7-439.6 \pm 10.1 \mu \mathrm{M}\right)$ 


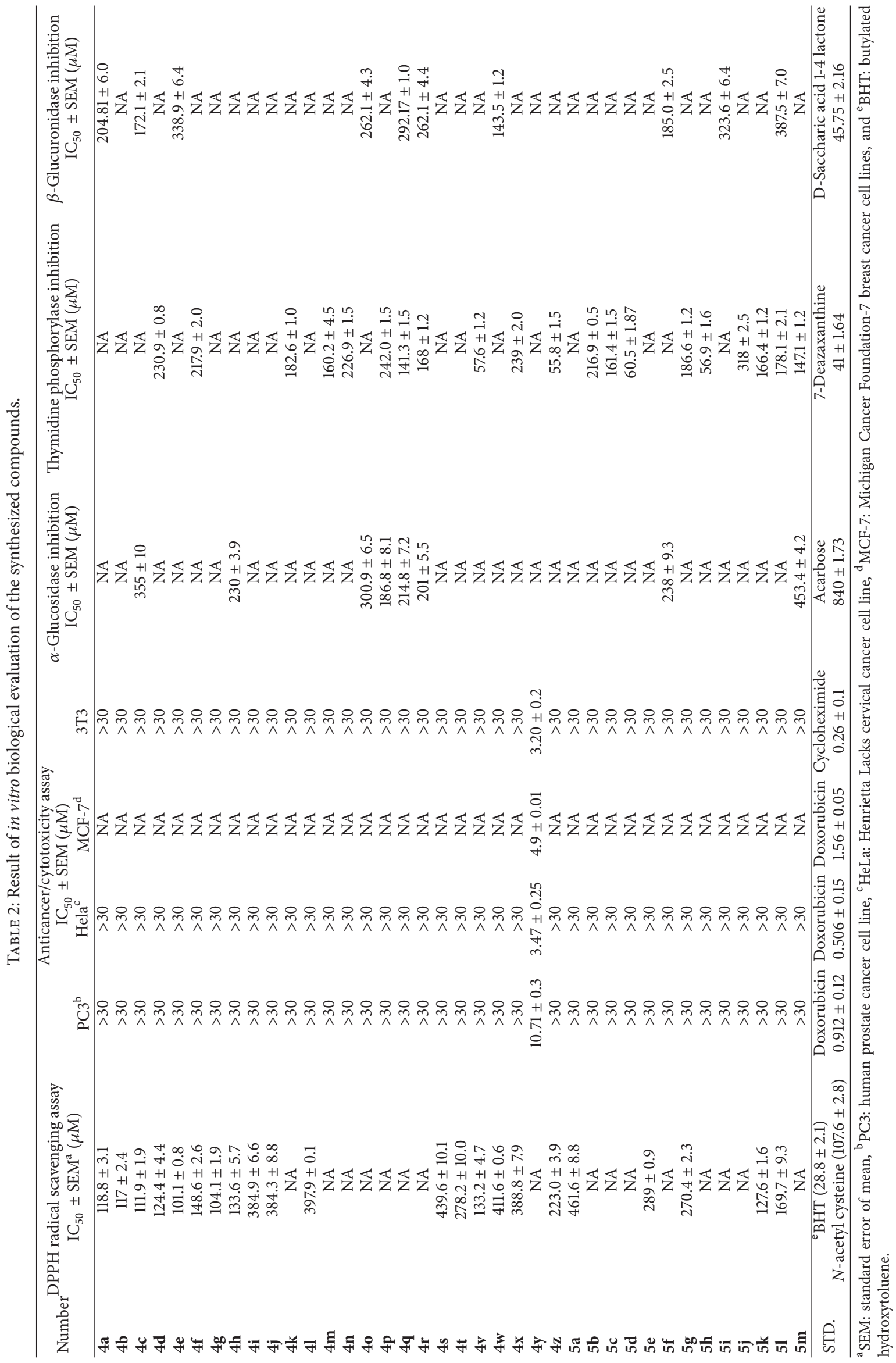


except compounds $\mathbf{4 m - 4 r}$ and $4 \mathbf{y}$ which were found to be inactive.

Similarly bis(2-hydroxy-4,4-dimethyl-6-oxocyclohex-1ene) $(\mathbf{5} \mathbf{a}-\mathbf{5 h})$ and 4-((6-hydroxy-1,3-dimethyl-2,4-dioxo1,2,3,4-tetrahydropyrimidin-5-yl) (6-hydroxy-2,4-dioxo1,2,3,4-tetrahydropyrimidin-5-yl)methyl) (5i-5k) rings containing compounds were also evaluated for their antioxidant potential by using DPPH radical scavenging assay. Parabromo-substituted phenyl ring containing compound 5k $\left(\mathrm{IC}_{50}=127.6 \pm 1.6 \mu \mathrm{M}\right)$ was found as a potent antioxidant agent against the tested standards $\mathrm{BHT}\left(\mathrm{IC}_{50}=\right.$ $128.8 \pm 2.1 \mu \mathrm{M})$. The compound showed similar antioxidant potential as BHT and showed a significant antioxidant agent against $N$-acetyl cysteine $\left(\mathrm{IC}_{50}=107.6 \pm 2.8 \mu \mathrm{M}\right)$. The gradual and drastic decrease in activity was for compounds 5l $\left(\mathrm{IC}_{50}=169.7 \pm 5.3 \mu \mathrm{M}\right), 5 \mathrm{~g}\left(\mathrm{IC}_{50}=270.4 \pm 2.3 \mu \mathrm{M}\right)$, $5 \mathbf{e}\left(\mathrm{IC}_{50}=289 \pm 0.9 \mu \mathrm{M}\right)$, and $5 \mathbf{a}\left(\mathrm{IC}_{50}=461.6 \pm 8.8 \mu \mathrm{M}\right)$ having para-methyl-, para-aldehyde-, ortho-chloro-, and ortho-nitro-substituted phenyl rings attached to the central barbiturate moieties. Compounds $\mathbf{5 b} \mathbf{- 5 d} \mathbf{d} \mathbf{5 f}$, and $\mathbf{5 h}-\mathbf{5 j}$ were found to be inactive.

On the basis of the observed antioxidant abilities of the above five different series of pyridinium adducts, it was concluded that bis(6-hydroxy-1,3-dimethyl-2,4-dioxo1,2,3,4-tetrahydropyrimidin-5-yl) ring containing compounds $\mathbf{4 a}-\mathbf{4 h}$ are the most active one. The activity may be due to the methyl substituted nitrogen atoms of the tetrahydropyrimidine rings that facilitate the electronic conjugation within the ring. However, the variation of antioxidant properties within the most important series is may be due to the presence of electron donating $\left(-\mathrm{OH}(\mathbf{4 f}),-\mathrm{OCH}_{3}(\mathbf{4 d})\right.$, and $\left.-\mathrm{CH}_{3}(\mathbf{4 b}, \mathbf{4 g})\right)$, electron withdrawing $\left(-\mathrm{NO}_{2}(\mathbf{4 a})\right.$ and -CHO (4c)), and halogen (-Br (4e)) substituents on the phenyl ring attached to the bis(6-hydroxy-1,3-dimethyl-2,4dioxo-1,2,3,4-tetrahydropyrimidin-5-yl) moiety. However, detailed studies are required to study the mechanism of action of these compounds.

3.3.2. Cytotoxic Activity. All synthesized diethyl ammonium salts of barbiturates $(\mathbf{4 a}-\mathbf{4 z}$ and $\mathbf{5 a}-\mathbf{5 m})$ were evaluated for their in vitro cytotoxicity against PC-3, HeLa, MCF-7 cancer, and 3T3 normal cell lines. All compounds were found to be noncytotoxic against PC-3, HeLa, MCF-7 cancer, and 3T3 cell lines except dichloro-substituted (2-hydroxy-4,4-dimethyl6-oxocyclohex-1-en-1-yl)-1,3-dimethyl-2,6-dioxo-1,2,3,6-tetrahydropyrimidin-4-olate ring containing compounds $4 \mathbf{y}$, which was found to be significantly toxic against all the cell lines $\left(\mathrm{PC} 3, \mathrm{IC}_{50}=10.71 \pm 0.26 \mu \mathrm{M}, \mathrm{HeLa}, \mathrm{IC}_{50}=3.47 \pm 0.3 \mu \mathrm{M}\right.$, MCF-7, $\mathrm{IC}_{50}=4.9 \pm 0.01 \mu \mathrm{M}$, and 3T3, $\left.\mathrm{IC}_{50}=3.20 \pm 0.2 \mu \mathrm{M}\right)$. Doxorubicin was used as a standard drug to compare the activities against PC3 $\left(\mathrm{IC}_{50}=1.70 \pm 0.29 \mu \mathrm{M}\right), \mathrm{HeLa}\left(\mathrm{IC}_{50}=\right.$ $0.51 \pm 0.15 \mu \mathrm{M})$, and MCF-3 $\left(\mathrm{IC}_{50}=0.92 \pm 0.01 \mu \mathrm{M}\right)$ cancer cell lines, while cycloheximide $\left(\mathrm{IC}_{50}=0.26 \pm 0.12 \mu \mathrm{M}\right)$ was used as a standard-in 3T3 cell line (Table 2).

3.3.3. $\alpha$-Glucosidase Inhibition Activity. Synthesized diethyl ammonium salts of barbiturates, $4 \mathbf{a}-\mathbf{4 z}$ and $5 \mathbf{a}-\mathbf{5 m}$, were also evaluated for their in vitro $\alpha$-glucosidase inhibition activity, in comparison to the standard drug, acarbose $\left(\mathrm{IC}_{50}=841 \pm\right.$
$1.73 \mu \mathrm{M})$. Among dimethyl ammonium salts (4a-4h), nitrosubstituted phenyl ring containing $4 \mathrm{c}\left(\mathrm{IC}_{50}=355 \pm 10 \mu \mathrm{M}\right)$ and naphthalene ring containing $4 \mathbf{h}\left(\mathrm{IC}_{50}=230 \pm 3.92 \mu \mathrm{M}\right)$ were found to be potent inhibitors of $\alpha$-glucosidase enzyme and showed more activity than acarbose $\left(\mathrm{IC}_{50}=841 \pm\right.$ $1.73 \mu \mathrm{M})$. All other compounds were found to be inactive.

Among diethyl ammonium salts having (2-hydroxy4,4-dimethyl-6-oxocyclohex-1-en-1-yl)-1,3-dimethyl-2,6dioxo-1,2,3,6-tetrahydropyrimidin-4-olate ring (4m-4z), para- $\mathrm{OCH}_{3}-\left(40, \mathrm{IC}_{50}=300.9 \pm 6.5 \mu \mathrm{M}\right)$, para-chloro$\left(4 \mathbf{p}, \mathrm{IC}_{50}=186.8 \pm 0.81 \mu \mathrm{M}\right)$, para-bromo- $\left(4 \mathbf{q}, \mathrm{IC}_{50}=\right.$ $214.8 \pm 0.72 \mu \mathrm{M})$, and meta-bromo- $\left(4 \mathrm{r}, \mathrm{IC}_{50}=201 \pm 5.5 \mu \mathrm{M}\right)$ substituted phenyl ring containing pyridinium adducts were found to be potent $\alpha$-glucosidase inhibitors and showed more activity than standard acarbose $\left(\mathrm{IC}_{50}=841 \pm 1.73 \mu \mathrm{M}\right)$. Other members of the series ( $4 m-4 n$ and $\mathbf{4 s}-\mathbf{4 z}$ ) were found to be inactive. The results showed the substitution effects of various functionalities of phenyl ring on the potential activity of tested series. The para-bromo-substituted phenyl ring containing compound $\mathbf{4 q}\left(\mathrm{IC}_{50}=214.8 \pm 0.72 \mu \mathrm{M}\right)$ showed more activity than tested standard. However, the replacement of bromo group with that of methyl, N,N-dimethyl, hydroxyl, and aldehyde functionalities resulted in complete loss of activity as observed in compounds $4 \mathbf{n}, \mathbf{4 t}, \mathbf{4 v}$, and $\mathbf{4 x}$, respectively. The presence of ortho-nitro-, ortho-choloro-, and para-choloro-substituted phenyl ring also contributed towards inactivity, as observed for compounds $\mathbf{4 t}, \mathbf{4 w}$, and $4 y$.

Similarly bis(2-hydroxy-4,4-dimethyl-6-oxocyclohex-1ene) ring containing diethyl ammonium pyridinium salts $(\mathbf{5 a}-\mathbf{5 h})$ were also evaluated for their in vitro $\alpha$-glucosidase enzyme inhibition activity. The series nitro-substituted phenyl ring containing $\mathbf{5 f}$ was found to be the only potent $\alpha$-glucosidase inhibitor. All other compounds (5a-5e and $5 \mathrm{~g}-5 \mathbf{h})$ were found to be inactive.

Among series of diethylaminium salts of pyridinium adducts (5i-5m) having 4-((6-hydroxy-1,3-dimethyl-2,4dioxo-1,2,3,4-tetrahydropyrimidin-5-yl) (6-hydroxy-2,4-dioxo-1,2,3,4-tetrahydropyrimidin-5-yl)methyl) central moiety attached with substituted phenyl ring, the benzaldehyde containing $5 \mathrm{~m}\left(\mathrm{IC}_{50}=453.4 \pm 4.2 \mu \mathrm{M}\right)$ was found to be the only potent inhibitor compound in comparison to against the standard drug acarbose $\left(\mathrm{IC}_{50}=841 \pm 1.73 \mu \mathrm{M}\right)$. All other compounds were found to be inactive. The results are summarized in Table 2.

3.3.4. Thymidine Phosphorylase Inhibition Activity. Five different series of diethyamonium salts of barbiturates, 4a$4 \mathrm{~g}, 4 \mathbf{i}-4 \mathrm{k}, 4 \mathrm{~m}-4 \mathrm{z}, 5 \mathrm{a}-\mathbf{5 h}$, and $5 \mathbf{i}-\mathbf{5 m}$, were also evaluated for their in vitro thymidine phosphorylase inhibition. 7-Deazaxanthine was used as a standard inhibitor $\left(\mathrm{IC}_{50}\right.$ $=41 \pm 1.46 \mu \mathrm{M})$. Phenol and naphthalene rings substituted (2-hydroxy-4,4-dimethyl-6-oxocyclohex-1-en-1-yl)1,3-dimethyl-2,6-dioxo-1,2,3,6-tetrahydropyrimidin-4-olate moiety containing compounds $4 \mathbf{v}\left(\mathrm{IC}_{50}=57.6 \pm 1.20 \mu \mathrm{M}\right)$ and $4 \mathbf{z}\left(\mathrm{IC}_{50}=55.8 \pm 1.50 \mu \mathrm{M}\right)$ were found to be the potent thymidine phosphorylase inhibitors. However, a drastic decrease in activity was observed when para-hydroxy group on phenyl ring was replaced with methyl $\left(\mathbf{4 n}, \mathrm{IC}_{50}\right.$ 
$=226.9 \pm 1.50 \mu \mathrm{M})$, chloro $\left(4 \mathbf{n}, \mathrm{IC}_{50}=242 \pm 1.5 \mu \mathrm{M}\right)$, bromo $\left(\mathbf{4 q}, \mathrm{IC}_{50}=141.3 \pm 1.5 \mu \mathrm{M}\right)$, and aldehyde $(\mathbf{4 x}$, $\left.\mathrm{IC}_{50}=239 \pm 2.0 \mu \mathrm{M}\right)$ groups, whereas the compounds 4o, 4s, 4t, 4w, and 4y have para-methoxy-, ortho-nitro-, ortho-N,N-dimethyl amine-, ortho-, para-dichloro-, and ortho-ortho-dichloro-substituted phenyl ring, respectively.

Among series of compounds $(\mathbf{5} \mathbf{a}-\mathbf{5 h})$ having bis(2hydroxy-4,4-dimethyl-6-oxocyclohex-1-ene) ring as central moiety, electron donating hydroxy- and methoxy-substituted phenyl ring containing compounds $5 \mathbf{h}\left(\mathrm{IC}_{50}=56.9 \pm 1.6 \mu \mathrm{M}\right)$ and $5 \mathrm{~d}\left(\mathrm{IC}_{50}=60.5 \pm 1.87 \mu \mathrm{M}\right)$, respectively, were found as potent inhibitors of thymidine phosphorylase enzyme against the tested standard 7-deazaxanthine which was $\left(\mathrm{IC}_{50}=41 \pm 1.46 \mu \mathrm{M}\right)$ used as standard. Again a drastic decrease in activity was observed for compounds $5 \mathbf{b}\left(\mathrm{IC}_{50}\right.$ $=216.9 \pm 0.5 \mu \mathrm{M}), 5 \mathrm{c}\left(\mathrm{IC}_{50}=161.4 \pm 1.5 \mu \mathrm{M}\right)$, and $5 \mathrm{~g}$ $\left(\mathrm{IC}_{50}=186.6 \pm 1.2 \mu \mathrm{M}\right)$ having meta-methyl-, ortho-methyl-, and para-aldehyde-substituted benzene ring respectively, instead of para-hydroxyl phenyl ring. The replacement of ortho-methyl with chloro-group makes the compound $\mathbf{5 e}$ completely inactive. Similarly the replacement of electron donating para-hydroxy-group of phenyl ring with electron withdrawing nitro-functionality also contributed towards inactivity of compound, as observed for $\mathbf{5 f}$. Phenol substituted bis(2-hydroxy-4,4-dimethyl-6-oxocyclohex-1-ene) ring containing $5 \mathbf{h}\left(\mathrm{IC}_{50}=56.9 \pm 1.6 \mu \mathrm{M}\right)$ was found to be the potent thymidine phosphorylase inhibitors.

All members of the series of diethylaminium salts of pyridinium adducts $(\mathbf{5 i}-\mathbf{5 m})$ having 4-((6-hydroxy-1,3-dimethyl2,4-dioxo-1,2,3,4-tetrahydropyrimidin-5-yl) (6-hydroxy-2,4dioxo-1,2,3,4-tetrahydropyrimidin-5-yl)methyl) central moiety attached with substituted phenyl ring were found to be weak inhibitors of thymidine phosphorylase enzyme against the tested standard compound 7-deazaxanthine $\left(\mathrm{IC}_{50}=41 \pm\right.$ $1.46 \mu \mathrm{M})$ with $\mathrm{IC}_{50}$ values $318 \pm 2.5 \mu \mathrm{M}, 166.4 \pm 1.2 \mu \mathrm{M}$, $178.1 \pm 2.1 \mu \mathrm{M}$, and $147.1 \pm 1.23 \mu \mathrm{M}$ for $5 \mathbf{j}, 5 \mathbf{k}, 5 \mathbf{l}$, and $5 \mathrm{~m}$, respectively, whereas para-chloro-substituted phenyl ring containing compound $\mathbf{5 i}$ was found to be inactive. The results are summarized in Table 1.

3.3.5. $\beta$-Glucuronidase Inhibition Activity. Compounds $4 \mathbf{a}-$ $4 \mathrm{~g}, 4 \mathbf{i}-4 \mathrm{k}, 4 \mathrm{~m}-4 \mathrm{z}, 5 \mathrm{a}-\mathbf{5 h}$, and $5 \mathbf{i}-\mathbf{5 m}$ were also evaluated for their in vitro $\beta$-glucuronidase inhibitory activity by using $\mathrm{D}$-saccharic acid 1,4-lactone as standard inhibitor $\left(\mathrm{IC}_{50}=\right.$ $45.75 \pm 2.16 \mu \mathrm{M})$. Compounds $4 \mathbf{a}\left(\mathrm{IC}_{50}=204.8 \pm 6.01 \mu \mathrm{M}\right)$, $4 \mathbf{c}\left(\mathrm{IC}_{50}=172.1 \pm 2.1 \mu \mathrm{M}\right), 4 \mathrm{e}\left(\mathrm{IC}_{50}=338.9 \pm 6.42 \mu \mathrm{M}\right), 4 \mathbf{o}$ $\left(\mathrm{IC}_{50}=262.1 \pm 4.35 \mu \mathrm{M}\right), \mathbf{4 q}\left(\mathrm{IC}_{50}=292.2 \pm 1.03 \mu \mathrm{M}\right), \mathbf{4 r}$ $\left(\mathrm{IC}_{50}=262.1 \pm 4.4 \mu \mathrm{M}\right), \mathbf{4 w}\left(\mathrm{IC}_{50}=143.5 \pm 1.24 \mu \mathrm{M}\right), \mathbf{5 f}\left(\mathrm{IC}_{50}\right.$ $=185 \pm 2.5 \mu \mathrm{M}), 5 \mathbf{i}\left(\mathrm{IC}_{50}=223.6 \pm 6.4 \mu \mathrm{M}\right)$, and $5 \mathbf{l}\left(\mathrm{IC}_{50}=\right.$ $387.5 \pm 7.0 \mu \mathrm{M})$ showed very weak $\beta$-glucuronidase inhibition potential, whereas pyridinium adducts $\mathbf{4 a - 4 c}, \mathbf{4 e}, \mathbf{4 g}-\mathbf{4 j}, \mathbf{4 l}$, $40,4 s-4 t, 4 w, 4 y, 5 a, 5 e-5 f$, and $5 i$ were found to be inactive (Table 2).

\subsection{Molecular Docking Studies}

3.4.1. Binding Interactions of $\alpha$-Glucosidase Enzyme. To define inhibitors interactions and their specificity, the docking study of the most active compound $\mathbf{4 p}$ against $\alpha$-glucosidase

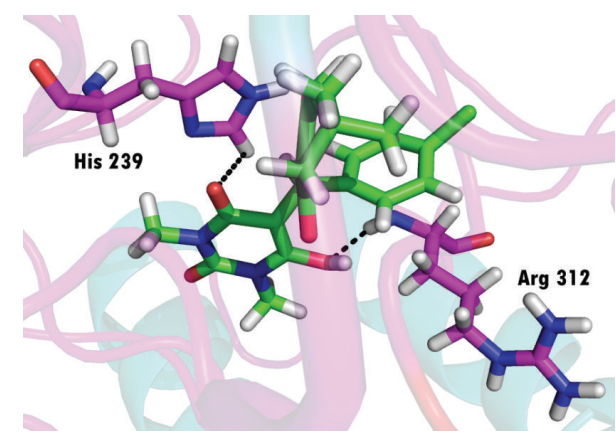

FIgURE 4: Three-dimensional representation of compound $\mathbf{4 p}$ with target macromolecule $\alpha$-glucosidase.

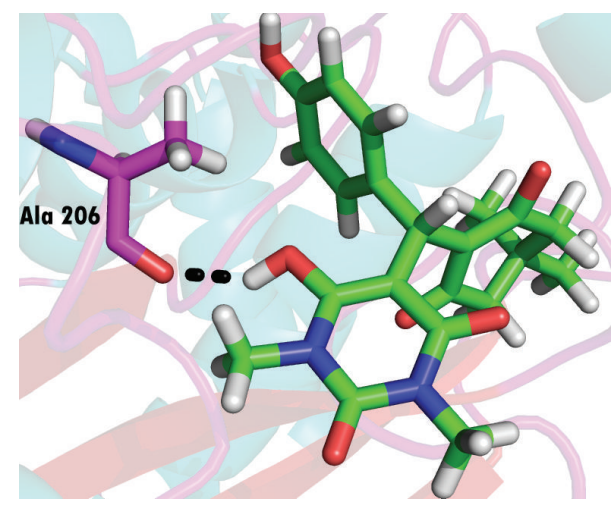

FIGURE 5: Three-dimensional representation of compound $\mathbf{4 v}$ with target macromolecule thymidine phosphorylase.

was carried out [23-25]. Ten different conformations of protein-ligand interactions were generated and the top ranked pose was selected to explore the binding interactions. In our docking study, we have observed two interactions with the active site residues of $\alpha$-glucosidase. The polar imidazole ring of His 239 showed hydrogen bond through its $\mathrm{H}$ atom with the lone pair of oxygen of the pyrimidinedione moiety of the compound, whereas Arg 312 formed hydrogen bond with the $\mathrm{OH}$ group of the same moiety of compound (Figure 4). The presence of polar and electron rich center in the form of pyrimidinedione and chlorobenzene groups of the compound was observed here as key factors for the interaction between compound and protein. A number of hydrogen bond donor and acceptor pairs with their suitable orientations were observed in the interaction pose. Furthermore, several hydrophobic interactions between the nonpolar active site residues and the compound $\mathbf{4 p}$ were also observed.

\subsubsection{Binding Interactions of Thymidine Phosphorylase} Enzyme. To explore the binding modes of these newly synthesized compounds in the active site of thymidine phosphorylase, the most active compound $\mathbf{4 v}$ was docked against this enzyme. The docking results showed that compound $4 \mathbf{v}$ well accommodated in the active site of thymidine phosphorylase (Figure 5). The active site residue Ala 206 established a hydrogen bond through its lone pair of 


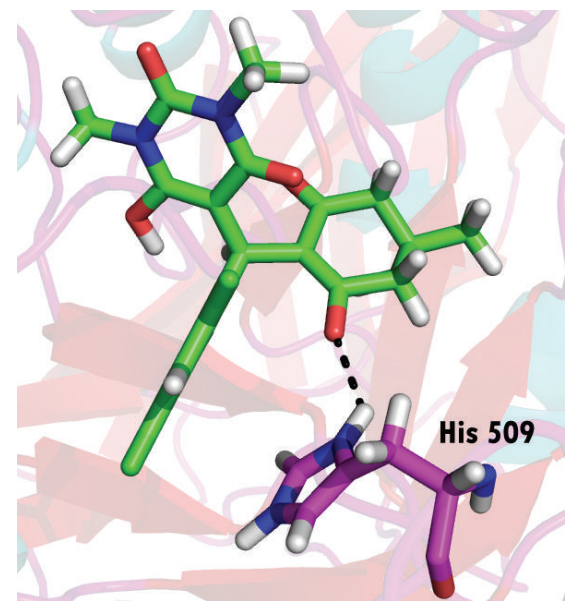

FIGURE 6: Three-dimensional representation of compound $4 \mathbf{w}$ with target enzyme $\beta$-glucuronidase.

oxygen with the active hydrogen of hydroxyl moiety of the pyrimidinedione group of the compound. However, the substituted phenolic group and the other electron rich centers of the pyrimidinedione showed poor behavior regarding interaction which may depend on the nature and orientation of the surrounding active site residues. Moreover, several weak hydrophobic interactions between the active site residues and the compound were also observed.

3.4.3. Binding Interaction of $\beta$-Glucuronidase Enzyme. The predicted binding mode of most active compound $\mathbf{4} \mathbf{w}$ against $\beta$-glucuronidase showed that a hydrogen bond is formed between the lone pair of oxygen of cyclohexanedione component of the compound and imidazole hydrogen of the important active site residue, His 509. An arene-arene and arenecation interaction was also observed between the pi electronic cloud of dichlorobenzene ring of compound and imidazole ring of His 509 (Figure 6). Besides hydrogen bonding and arene-arene interactions, several hydrophobic interactions between compound $\mathbf{4 w}$ and the active site residues were also observed. The pyrimidinedione moiety in this case was observed to be passive regarding interactions.

\section{Conclusions}

In conclusion, barbiturate salts were synthesized by one-pot multicomponent reactions via tandem Aldol/Michael addition reactions between barbituric acid and dimedone with aldehydes, mediated by aqueous diethylamine. The molecular structures of $\mathbf{5 a}, \mathbf{5 d}$, and $\mathbf{5 f}$ were deduced by singlecrystal X-ray diffraction techniques. All of the synthesized compounds were evaluated for their antioxidant potential in vitro by using DPPH radical scavenging assay as well as enzyme inhibition activities against $\alpha$-glucosidase, thymidine phosphorylase, and $\beta$-glucuronidase enzymes. Compounds $4 \mathbf{a}-4 \mathrm{~g}\left(\mathrm{IC}_{50}=101.8 \pm 0.8-124.4 \pm 4.4 \mu \mathrm{M}\right)$ were found to be the potent antioxidants as compared to the standards, BHT $\left(\mathrm{IC}_{50}=128.8 \pm 2.1 \mu \mathrm{M}\right)$, and $N$-acetylcysteine $\left(\mathrm{IC}_{50}=\right.$ $107.6 \pm 2.8 \mu \mathrm{M})$. Compound $\mathbf{4 p}\left(\mathrm{IC}_{50}=186.8 \pm 8.1 \mu \mathrm{M}\right)$ was found to be a potent $\alpha$-glucosidase inhibitor. Compound $\mathbf{4 v}$ $\left(\mathrm{IC}_{50}=8.7 \pm 1.2 \mu \mathrm{M}\right)$ was found to be a potent thymidine phosphorylase inhibitor. Promising results indicate that these compounds need to be investigated as antioxidant agents to treat various associated oxidative disorders. Similarly their enzyme inhibitory potential can be reported on drug discovery program.

\section{Competing Interests}

The authors declare that there are no competing interests regarding the publication of this paper.

\section{Authors' Contributions}

Assem Barakat, Hazem A. Ghabbour, Abdullah Mohammed Al-Majid, Qurat-ul-ain, Rehan Imad, Kulsoom Javaid, Nimra Naveed Shaikh, Sammer Yousuf, M. Iqbal Choudhary, and Abdul Wadood contributed equally to this work.

\section{Acknowledgments}

The authors would like to extend their sincere appreciation to the Deanship of Scientific Research at King Saud University for funding this Research Group no. (RGP-257-1435-1436).

\section{References}

[1] K. Undheim, T. Bennecke, A. R. Katritzky, C. W. Rees, and E. F. V. Scriven, Comprehensive Heterocyclic Chemistry, vol. 6, supplement 93, Elsevier Pergamon, Oxford, UK, 1996.

[2] B. Taylor, Modern Medical Chemistry, Prentice Hall, New York, NY, USA, 1994.

[3] R. Y. Levina and F. K. Velichko, "Advances in the chemistry of barbituric acids," Russian Chemical Reviews, vol. 29, no. 8, pp. 437-459, 1960.

[4] P. Sing and K. Paul, "A simple synthesis of 5-spirobarbituric acids and transformations of spirocyclopropane barbiturates to 5 -substitutated barbiturates," Indian Journal of Chemistry B, vol. 45, pp. 247-251, 2006.

[5] X. Chen, K. Tanaka, and F. Yoneda, "Simple new method for the synthesis of 5-deaza-10-oxaflavin, a potential organic oxidant," Chemical and Pharmaceutical Bulletin, vol. 38, no. 2, pp. 307311, 1990 .

[6] L. R. Morgan, B. S. Jursic, C. L. Hooper, D. M. Neumann, K. Thangaraj, and B. LeBlanc, "Anticancer activity for 4, 4'-dihydroxybenzophenone-2, 4-dinitrophenylhydrazone (A-007) analogues and their abilities to interact with lymphoendothelial cell surface markers," Bioorganic \& Medicinal Chemistry Letters, vol. 12, no. 23, pp. 3407-3411, 2002.

[7] G. Orzalesi, P. Gratteri, and S. Selleri, "Synthesis of new 1,3dicyclohexyl barbituric acid derivatives with anti-inflammatory potential activity," European Journal of Medicinal Chemistry, vol. 25, no. 2, pp. 197-201, 1990.

[8] D. T. Puerta and S. M. Cohen, "A bioinorganic perspectives on matrix metalloproteinase inhibition," Current Topics in Medicinal Chemistry, vol. 4, no. 15, pp. 1551-1573, 2004.

[9] M. E. Wolff, Burger's Medicinal Chemistry and Drug Discovery, John Wiley \& Sons, New York, NY, USA, 1997. 
[10] E. Fischer and J. von Mering, "Ueber eine neue klasse von schlafmitteln," in Untersuchungen aus Verschiedenen Gebieten, M. Bergmann, Ed., pp. 671-679, Springer, Berlin, Germany, 1924.

[11] W. J. Doran, "Barbituric acid hypnotics," Medicinal Chemistry, vol. 4, pp. 164-167, 1959.

[12] B. Bobranski, "Progress in chemistry of barbituric acid," Wiadomości Chemiczne, vol. 31, pp. 231-278, 1977.

[13] S. Senda, H. Izumi, and H. Fujimura, "Uracil derivaives and related compounds. 6. Derivatives of 5-alkyle-2,4,6-trioxoperhydropyrimidine as antiphlogistics," Arzneimittel Forschung, vol. 17, no. 12, p. 151, 1967.

[14] N. Moussier, L. Bruché, F. Viani, and M. Zanda, "Fluorinated barbituric acid derivatives: synthesis and bio-activity," Current Organic Chemistry, vol. 7, no. 11, pp. 1071-1080, 2003.

[15] P.-G. Pietta, "Flavonoids as antioxidants," Journal of Natural Products, vol. 63, no. 7, pp. 1035-1042, 2000.

[16] N. Ramarathnam, T. Osawa, H. Ochi, and S. Kawakishi, "The contribution of plant food antioxidants to human health," Trends in Food Science \& Technology, vol. 6, no. 3, pp. 75-82, 1995.

[17] A. Barakat, A. M. Al-Majid, H. J. Al-Najjar et al., "Zwitterionic pyrimidinium adducts as antioxidants with therapeutic potential as nitric oxide scavenger," European Journal of Medicinal Chemistry, vol. 84, pp. 146-154, 2014.

[18] P. Singh, M. Kaur, and P. Verma, "Design, synthesis and anticancer activities of hybrids of indole and barbituric acidsIdentification of highly promising leads," Bioorganic and Medicinal Chemistry Letters, vol. 19, no. 11, pp. 3054-3058, 2009.

[19] A. M. Al-Majid, A. Barakat, H. J. Al-Najjar, Y. N. Mabkhot, H. A. Ghabbour, and H.-K. Fun, "Tandem aldol-michael reactions in aqueous diethylamine medium: a greener and efficient approach to bis-pyrimidine derivatives," International Journal of Molecular Sciences, vol. 14, no. 12, pp. 23762-23773, 2013.

[20] A. Barakat, A. M. Al-Majid, G. Lotfy et al., "Synthesis and dynamics studies of barbituric acid derivatives as urease inhibitors," Chemistry Central Journal, vol. 9, no. 1, article 63, 2015.

[21] G. M. Sheldrick, "A short history of SHELX," Acta Crystallographica A, vol. 64, pp. 112-122, 2008.

[22] A. L. Spek, "Structure validation in chemical crystallography," Acta Crystallographica Section D: Biological Crystallography, vol. 65, no. 2, pp. 148-155, 2009.

[23] F. Rahim, K. Ullah, H. Ullah et al., "Triazinoindole analogs as potent inhibitors of $\alpha$-glucosidase: synthesis, biological evaluation and molecular docking studies," Bioorganic Chemistry, vol. 58, pp. 81-87, 2015.

[24] F. Rahim, F. Malik, H. Ullah et al., "Isatin based Schiff bases as inhibitors of $\alpha$-glucosidase: synthesis, characterization, in vitro evaluation and molecular docking studies," Bioorganic Chemistry, vol. 60, article 1803, pp. 42-48, 2015.

[25] A. Barakat, A. M. Al-Majid, M. S. Islam et al., "Molecular structure investigation and biological evaluation of Michael adducts derived from dimedone," Research on Chemical Intermediates, vol. 42, pp. 4041-4053, 2016. 

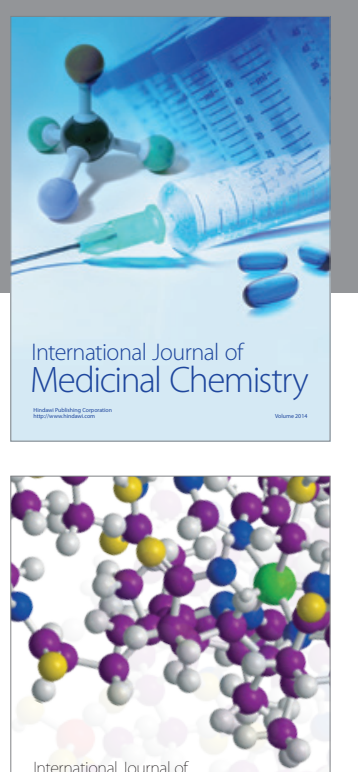

Carbohydrate Chemistry

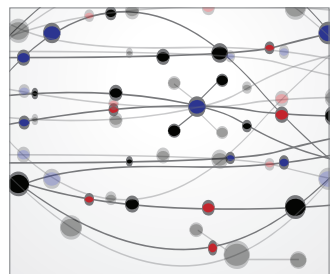

The Scientific World Journal
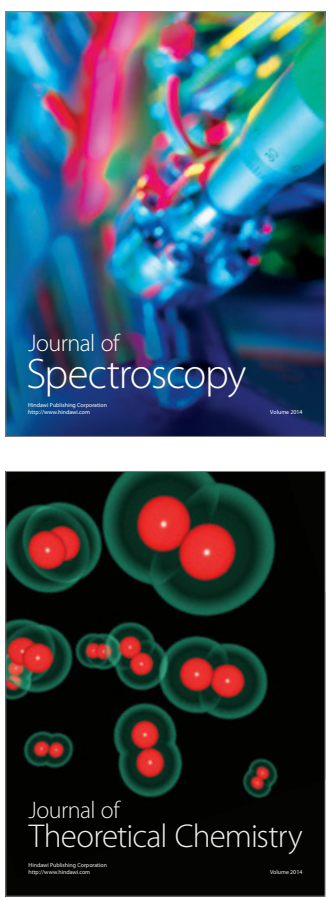
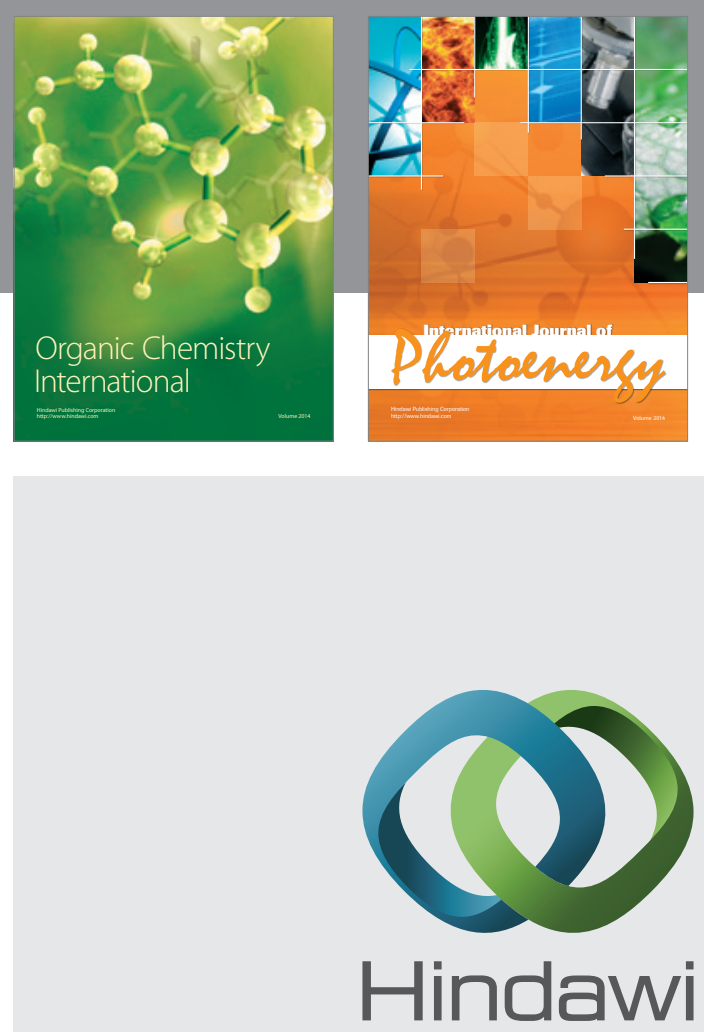

Submit your manuscripts at

http://www.hindawi.com

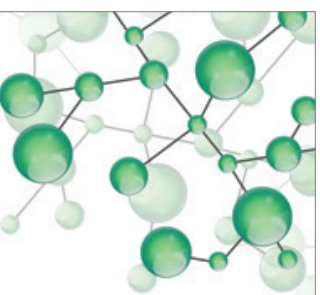

International Journal of

Inorganic Chemistry

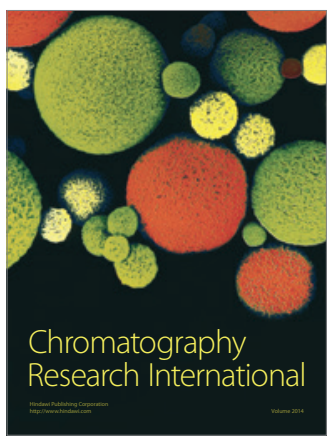

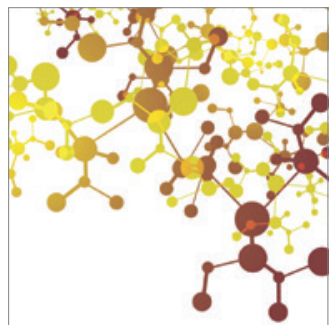

Applied Chemistry
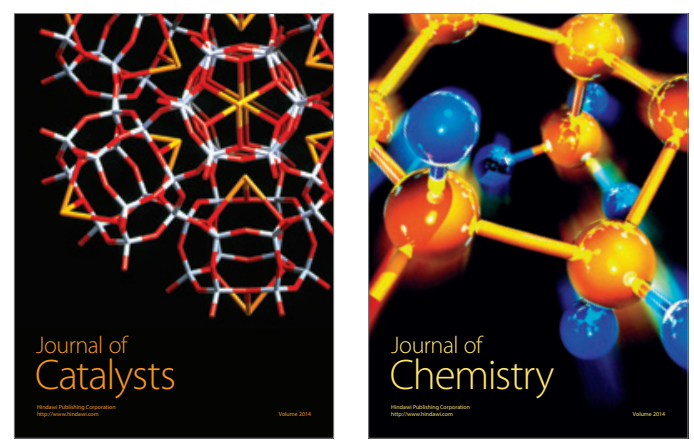
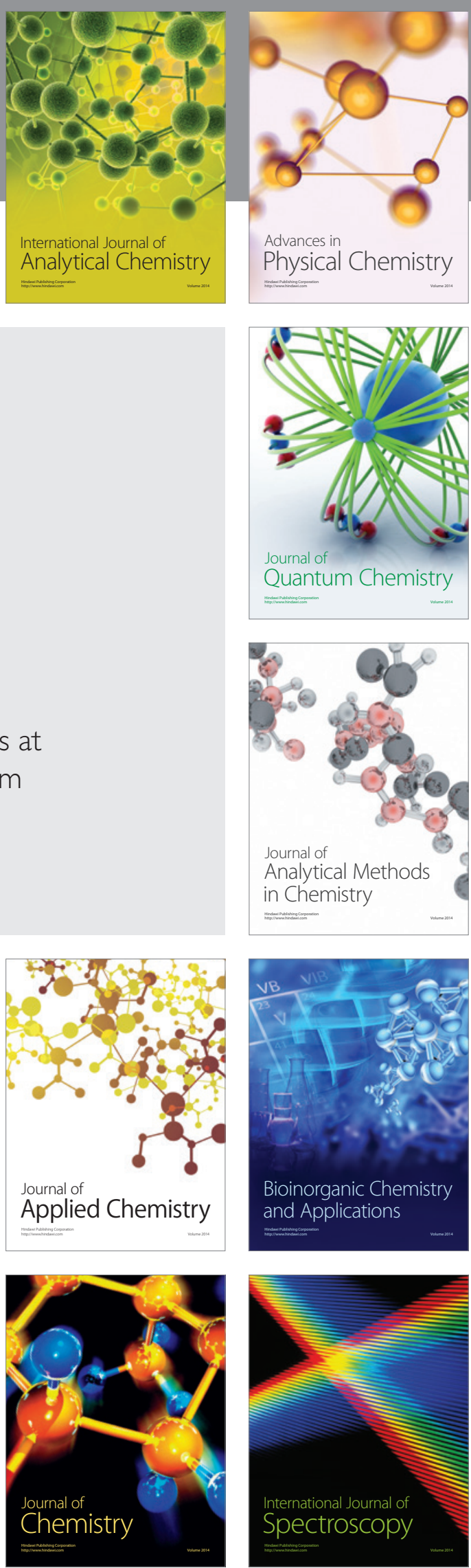